\title{
Proposed Heuristic Method for Solving Assignment Problems
}

\author{
S. K. Amponsah1, D. Otoo², S. Salhi' ${ }^{3}$, E. Quayson ${ }^{3}$ \\ ${ }^{1}$ Department of Mathematics, Kwame Nkrumah University of Science and Technology, Kumasi, Ghana \\ ${ }^{2}$ Department of Mathematics and Statistics, University of Energy and Natural Recourses, Sunyani, Ghana \\ ${ }^{3}$ Management Science Group, University of Kent, Kent, UK \\ Email: dominicotoo@rocketmail.com
}

How to cite this paper: Amponsah, S.K., Otoo, D., Salhi, S. and Quayson, E. (2016) Proposed Heuristic Method for Solving Assignment Problems. American Journal of Operations Research, 6, 436-441.

http://dx.doi.org/10.4236/ajor.2016.66040

Received: July 24, 2016

Accepted: November 11, 2016

Published: November 14, 2016

Copyright $\odot 2016$ by authors and Scientific Research Publishing Inc. This work is licensed under the Creative Commons Attribution International License (CC BY 4.0).

http://creativecommons.org/licenses/by/4.0/

\begin{abstract}
Assignment of jobs to workers, contract to contractors undergoing a bidding process, assigning nurses to duty post, or time tabling for teachers in school and many more have become a growing concern to both management and sector leaders alike. Hungarian algorithm has been the most successful tool for solving such problems. The authors have proposed a heuristic method for solving assignment problems with less computing time in comparison with Hungarian algorithm that gives comparable results with an added advantage of easy implementation. The proposed heuristic method is used to compute some bench mark problems.
\end{abstract}

\section{Keywords}

Assignment Problem, Hungarian Algorithm, Heuristic

\section{Introduction}

The Assignment Problem (AP), also known as the maximum weighted bipartite matching problem, is a special type of Linear Programming Problem (LPP), in which the objective is to assign number of jobs to number of workers at a minimum cost (time). The mathematical formulation of the problem suggests that this is an integer programming problem and is highly degenerated. All the algorithms developed to find optimal solution of transportation problem are applicable to assignment problem. However, due to its highly degeneracy nature, a specially designed algorithm widely known as Hungarian method proposed by Kuhn [1] is used for its solution. The assignment problem, also known as the maximum weighted bipartite matching problem, 
is a widely-studied problem applicable to many domains, specifically for maximization of output or minimization of cost. It can be stated as follows: given a bipartite graph made up of two partitions $V$ and $U$, and a set of weighted edges $E$ between the two partitions, the problem requires the selection of a subset of the edges with a maximum sum of weights such that each node $u_{i} \in V$ or $u_{i} \in U$ is connected to at most one edge. The problem may also be phrased as a minimization problem by considering, instead of edge weights $w_{i j}$, a set of non-negative edge costs, $c_{i j}=W-w_{i j}$, where $W$ is at least as large as the maximum of all the edge weights. It can also be stated as: how to determine the best possible assignment of workers to jobs, such that the total ratings are maximized [2].

\section{Related Works}

Franses and Gerhard [3] studied an assignment problem particular to the personnel scheduling of organisations such as laboratories. Here the authors have to assign tasks to employees. The authors focused on the situation where this assignment problem reduces to constructing maximal matchings in a set of interrelated bipartite graphs. The authors described in detail how the continuity of tasks over the week is achieved to suit the wishes of the planner. Turkish Armed Forces assign thousands of personnel to vacant positions every year, and want to utilize the personnel to a maximum extent by assigning the right person to the right job, while taking into consideration the hierarchy constraint, Cimen [4].

Toroslu and Arslanoglu [5], presented variations of the standard assignment problem with matching constraints by introducing structures in the partitions of the bipartite graph, and by defining constraints on these structures.

Naveh et al. [6] presented a novel solution designed to bridge the gap between the need for high-quality matches and the need for timeliness. By applying constraint programming, a subfield of artificial intelligence, the authors dealt successfully with the complex constraints encountered in the field and reach near-optimal assignments that take into account all resources and positions in the pool.

Katta and Jay [7] presented the problem of allocating a set of indivisible objects to agents in a fair and efficient manner.

Bogomolnaia and Moulin [8] considered the case in which all agents have strict preferences, and proposed the Probabilistic Serial (PS) mechanism; they defined a new notion of efficiency, called ordinal efficiency, and prove that the probabilistic serial mechanism finds an envy-free ordinarily efficient assignment.

Zhang and Bard [9] studied a multi-period assignment problem that arises as part of a weekly planning problem at mail processing and distribution centres.

\subsection{The Assignment Problem}

Suppose we have $n$ resources to which we want to assign to $n$ tasks on a one-to-one basis. Suppose also that we know the cost of assigning a given resource to a given task. We wish to find an optimal assignment-one which minimizes total cost. 


\subsection{Mathematical Model}

Let $C_{i j}$ be the cost of assigning the $i$ th resource to the th task. We define the cost matrix to be the $n \times n$ matrix

Job 1 Job 2 Job $3 \quad \cdots \quad$ Job $j \quad \cdots \quad$ Job $n$

\begin{tabular}{|c|c|c|c|c|c|c|}
\hline Worker 1 & $C_{11}$ & $C_{11}$ & $C_{11}$ & $\cdots$ & $C_{1 j}$ & $\cdots$ \\
\hline$C_{i j}=$ Worker 2 & $C_{21}$ & $C_{22}$ & $C_{23}$ & $\ldots$ & $C_{2 j}$ & \\
\hline Worker $i$ & $C_{i 1}$ & $C_{i 2}$ & $C_{i 3}$ & & $C_{i j}$ & \\
\hline Worker $N$ & $C_{n 1}$ & $C$ & & & $C_{n i}$ & $\ldots$ \\
\hline
\end{tabular}

An assignment is a set of $n$ entry positions in the cost matrix, no two of which lie in the same row or column. The sum of the $n$ entries of an assignment is its cost. An assignment with the smallest possible cost is called an optimal assignment. The assignment problem can be written mathematically as:

Minimize

$$
\begin{aligned}
& Z=\sum_{i=1}^{n} \sum_{j=1}^{n} C_{i j} x_{i j} \\
& \text { subject to } \\
& \sum_{i=1}^{n} x_{i j}=1 \\
& \sum_{j=1}^{n} x_{i j}=1 \quad x_{i j} \in[0,1] \\
& x_{i j}= \begin{cases}1 ; \text { if the } i \text { th worker is assigned the } j \text { th job } \\
0 ; & \text { otherwise }\end{cases}
\end{aligned}
$$

\subsection{Hungarian Method}

The following algorithm applies the above theorem to a given $n \times n$ cost matrix to find an optimal assignment.

Step 1: Subtract the smallest entry in each row from all the entries of its row.

Step 2: Subtract the smallest entry in each column from all the entries of its column.

Step 3: Draw lines through appropriate rows and columns so that all the zero entries of the cost matrix are covered and the minimum number of such lines is used.

Step 4: Test for Optimality:

i) If the minimum number of covering lines is $n$, an optimal assignment of zeros is possible and we are finished.

ii) If the minimum number of covering lines is less than $n$, an optimal assignment of zeros is not yet possible. In that case, proceed to Step 5.

Step 5: Determine the smallest entry not covered by any line. Subtract this entry from each uncovered row, and then add it to each covered column. Return to Step 3.

\subsection{Penalty method (Proposed Heuristic Method)}

The algorithm for $n \times n$ cost matrix using the proposed heuristic method is as shown below. 
- Compute row/column penalties by subtracting the least entry from the next least;

- Select the two maximum penalties from the row or column penalties. Use the maximum penalties to select the least cost among these penalties.

- Cross-out the row and column on which the minimum cost is located

- If there is a tie of the minimum cost from the same row or the same column, choose the cost of the third maximum penalty

- Break ties arbitrary when there is a tie on the cost from maximum row and column penalty

- If the least cost emanating from maximum penalty has its corresponding row or column penalty as zero, choose the least cost from the next maximum penalty.

- Repeat bullet two

- Stopping criteria: stop when all rows and columns have been crossed out.

\section{Comparative Results Using the Hungarian and the Proposed Method}

The following examples give the optimal results from the two methods and their computing time. The two algorithms were coded using $\mathrm{C}++$ on

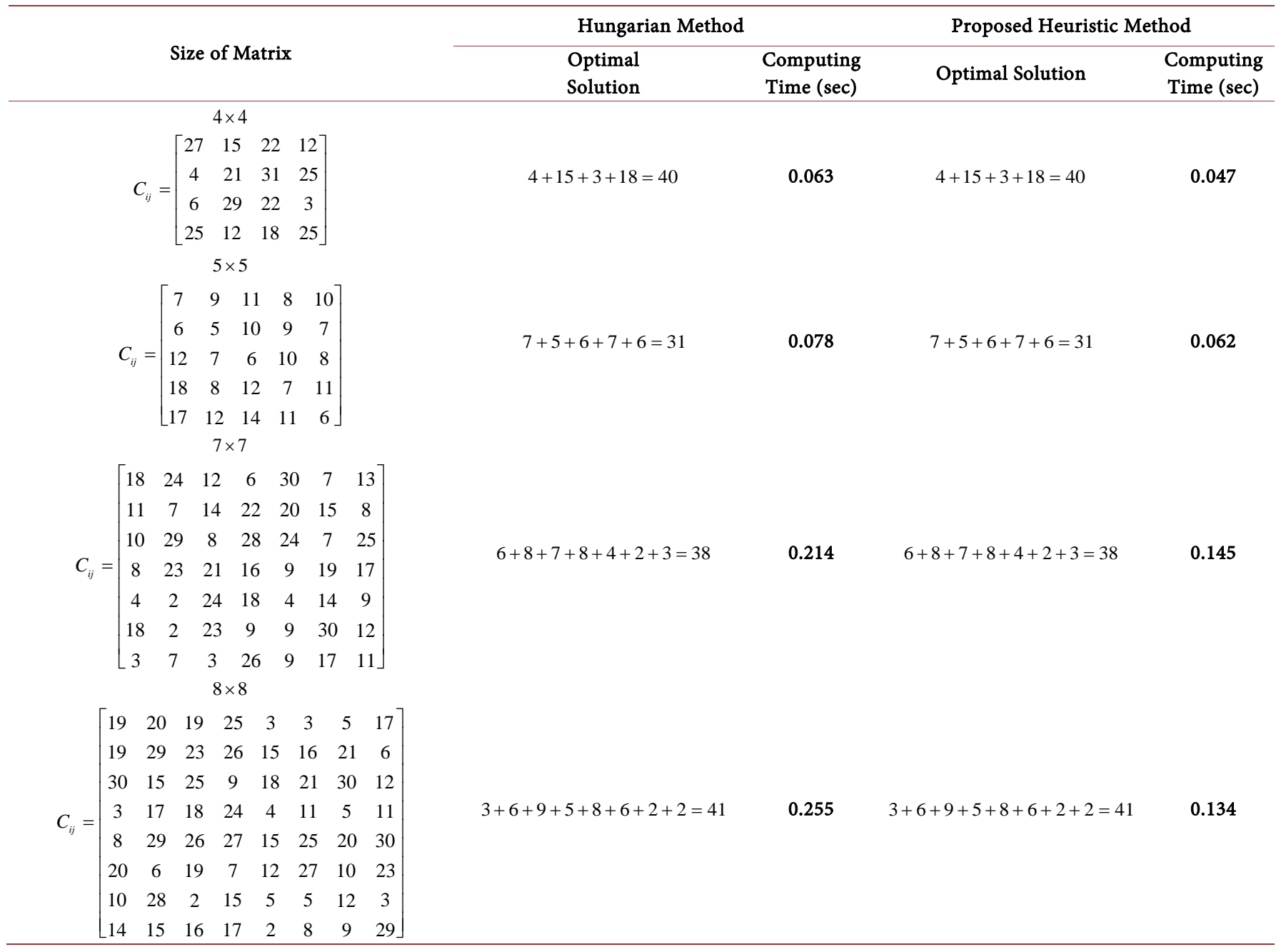




\section{Continued}

$$
C_{i j}=\left[\begin{array}{ccccccccc}
7 & 9 & 11 & 8 & 10 & 12 & 15 & 13 & 14 \\
6 & 5 & 10 & 9 & 7 & 11 & 13 & 18 & 15 \\
12 & 7 & 6 & 10 & 8 & 9 & 12 & 17 & 13 \\
18 & 8 & 12 & 7 & 11 & 10 & 14 & 16 & 19 \\
17 & 12 & 14 & 11 & 6 & 8 & 10 & 15 & 18 \\
14 & 9 & 13 & 12 & 5 & 7 & 8 & 19 & 16 \\
8 & 10 & 15 & 10 & 12 & 18 & 17 & 20 & 19 \\
11 & 13 & 16 & 17 & 14 & 15 & 21 & 12 & 17 \\
15 & 14 & 9 & 16 & 13 & 6 & 20 & 21 & 11
\end{array}\right]
$$

$10 \times 10$

$C_{i j}=\left[\begin{array}{cccccccccc}14 & 13 & 16 & 26 & 12 & 27 & 13 & 24 & 16 & 18 \\ 12 & 8 & 20 & 25 & 22 & 27 & 16 & 29 & 30 & 11 \\ 31 & 29 & 5 & 16 & 19 & 12 & 14 & 8 & 7 & 17 \\ 16 & 14 & 5 & 28 & 14 & 25 & 19 & 7 & 24 & 5 \\ 3 & 24 & 9 & 17 & 17 & 8 & 27 & 22 & 11 & 3 \\ 2 & 8 & 8 & 23 & 29 & 31 & 18 & 18 & 13 & 6 \\ 2 & 29 & 27 & 20 & 26 & 16 & 2 & 18 & 22 & 28 \\ 26 & 28 & 15 & 3 & 10 & 24 & 21 & 14 & 27 & 3 \\ 12 & 6 & 22 & 17 & 28 & 15 & 30 & 19 & 24 & 25 \\ 5 & 11 & 4 & 15 & 29 & 21 & 4 & 2 & 14 & 19\end{array}\right]$

\section{Results and Conclusion}

Test computation from a randomly generated numbers with our proposed heuristic method gave comparable results compared with that of Hungarian method. However, the computational time and convergence rate of the proposed method showed much less time than that of the Hungarian method.

\section{References}

[1] Bogomolnaia, A. and Moulin, H. (2001) New Solution to the Random Assignment Problem. Journal of Economic Theory, 100, 295-328. http://dx.doi.org/10.1006/jeth.2000.2710

[2] Cimen, Z. (2001) A Multi-Objective Decision Support Model for the Turkish Armed Forces Personnel Assignment System. Department of Operational Sciences, Air Force Institute of Technology, Ohio.

[3] Franses, P. and Gerhard, P. (2003) Personnel Scheduling in Laboratories. In: Burke, E. and De Causmaecker, P., Eds., Practice and Theory of Automated Timetabling IV, Vol. 2740, Lecture Notes in Computer Science, Springer Berlin, Heidelberg, 113-119.

[4] Katta and Jay (2005) Fair and Efficient Assignment via the Probabilistic Serial Mechanism.

[5] Kuhn, H.W. (1955) The Hungarian Method for the assignment problem. Naval Research Logistics Quarterly, 2, 83-97. http://dx.doi.org/10.1002/nav.3800020109 
[6] Munkres, J. (1957) Algorithms for the Assignment and Transportation Problems. Journal of the Society for Industrial and Applied Mathematics, 5, 32-38.

http://dx.doi.org/10.1137/0105003

[7] Naveh, Y., Richter, Y., Altshuler, Y., Gresh, D.L. and Connors, D.P. (2007) Workforce Optimization: Identification and Assignment of Professional Workers Using Constraint Programming. IBM Journal of Research and Development, Business Optimization Archive, 51, 263-279. http://dx.doi.org/10.1147/rd.513.0263

[8] Yilmaz, A. and Toroslu, I.H. (2011) Adaptation and Fine-Tuning of the Weighted Sum Method on Personnel Assignment Problem with Hierarchical Ordering and Team Constraints. 26th International Symposium on Computer and Information Sciences, London, 26-28 September 2011, 571-576.

[9] Zhang, X. and Bard, J.F. (2005) Equipment Scheduling at Mail Processing and Distribution Centers. IIE Transactions, 37, 175-187. http://dx.doi.org/10.1080/07408170590885657

\section{Submit or recommend next manuscript to SCIRP and we will provide best service} for you:

Accepting pre-submission inquiries through Email, Facebook, LinkedIn, Twitter, etc. A wide selection of journals (inclusive of 9 subjects, more than 200 journals)

Providing 24-hour high-quality service

User-friendly online submission system

Fair and swift peer-review system

Efficient typesetting and proofreading procedure

Display of the result of downloads and visits, as well as the number of cited articles

Maximum dissemination of your research work

Submit your manuscript at: http://papersubmission.scirp.org/

Or contact ajor@scirp.org 